\title{
Decomposition Procedure Using Methyl Orthoformate to Analyze Silicone Polymers
}

\author{
Yuichiro FuJImoto, ${ }^{* \dagger}$ Keisuke Sogabe, ${ }^{*}$ and Hajime OHTAnI** \\ *Kaneka Techno Research Corporation, 5-1-1 Torikainishi, Settsu, Osaka 566-0072, Japan \\ **Department of Materials Science and Engineering, Graduate School of Engineering, Nagoya Institute of \\ Technology, Gokiso, Showa, Nagoya, Aichi 466-8555, Japan
}

\begin{abstract}
A new decomposition method for structural analysis of polysiloxanes (silicones) was developed using methyl orthoformate. The siloxane bonds in samples with vinyl and/or methyl side groups decomposed under relatively mild acidic conditions up to around $70^{\circ} \mathrm{C}$ and were followed by methoxylation at the cleaved linkages with few side reactions. The product yields with respect to the siloxane monomer units were 98 - 100\% for low molecular weight model siloxane compounds. Additionally, this method decomposed the silicone polymer sample in a similar manner with decomposition yields of 98 and $103 \%$ for the dimethylsiloxane main chain and dimethylvinylsilyl end groups, respectively. These results demonstrate that the proposed decomposition method should be an effective pretreatment procedure for structural and compositional analyses of silicone polymers.
\end{abstract}

Keywords Polysiloxane, methyl orthoformate, decomposition, GC/MS

(Received February 12, 2014; Accepted May 7, 2014; Published July 10, 2014)

\section{Introduction}

Silicone is an important material in a variety of fields due to its excellent heat resistance, weather resistance, and chemical stability of the siloxane bond in the backbone. Depending on the molecular weight, silicone shows diverse properties ranging from liquid to solid. Furthermore, organic functional groups in the side chain allow unique surface properties (e.g., hydrophobicity or hydrophilicity) beyond inorganic silicon materials. Therefore, the structure of silicone compounds (e.g., these functional groups) often needs to be analyzed in detail. If the compound is soluble in an appropriate solvent, it is possible to analyze the functional groups and the molecular weight, e.g. by nuclear magnetic resonance (NMR) and size exclusion chromatography (SEC), respectively. However, when it is insoluble due to a crosslinked structure or high molecular weight, the limited methods for analysis make a detailed structural analysis quite difficult, although infrared absorption spectrometry (IR) can provide rough functional group information.

Meanwhile, pyrolysis GC/MS has been reported as a possible method to analyze silicone, even if the sample is insoluble in any solvent. ${ }^{1-4}$ However, polysiloxane thermally decomposes into cyclic oligosiloxane by siloxane exchange reactions. Therefore, pyrolysis GC/MS analysis provides partial information about the original molecular structure of the polysiloxane, but obtaining the molecular weight information is difficult. Furthermore, the structures of cyclic oligosiloxanes produced by pyrolysis are diverse in accordance with the type of

† To whom correspondence should be addressed.

E-mail: Yuichiro.Fujimoto@kaneka.co.jp side chain functional groups. Consequently, a high level of skill is often required to identify the original detailed structures of silicone polymers on the basis of the observed pyrograms. Moreover, since thermal decomposition during pyrolysis GC/ MS analysis produces cyclic siloxane oligomers in various sizes, it is not possible to obtain the exact composition of the original silicone samples.

Preprocessing methods to decompose a silicone sample into its constituent monomers quantitatively and selectively are essential for its accurate compositional analysis. Chemical decomposition methods to analyze silicone reported to date include ethoxysilane degradation, ${ }^{5,6}$ fluorosilane degradation, ${ }^{7}$ alkali fusion method, ${ }^{8,9}$ etc. The ethoxysilane degradation method decomposes the siloxane bond by heating through the reaction under alkaline conditions with the addition of tetraethoxysilane to the silicone samples. However, it is impossible to analyze accurately the hydrosiloxane content in the sample from the generated decomposition product because the hydrosilyl groups are methoxylated during the ethoxysilane degradation processing. Additionally, this decomposition method is not suitable as a pretreatment for quantitative analysis because all siloxane bonds are not necessarily cleaved.

Similarly to the ethoxysilane degradation, the fluorosilylation degradation method is not suitable for quantitative analysis. The fluorosilylation method generally requires extremely harsh conditions in order for decomposition to proceed. In addition, the side chain phenyl groups in the silicone samples are eliminated because they react with boron trifluoride during the processing. Although the alkali fusion method can provide some functional group information, it is difficult to obtain detailed information about the structure and composition of the silicone sample because the siloxane bond cannot be decomposed by this method. Consequently, the pretreatment methods 
(A)<smiles>C[Si](C)(C)O[Si](C)(C)C</smiles>

(B)<smiles>C=C[Si](C)(C)O[Si](C)(C)CC</smiles>

(C)

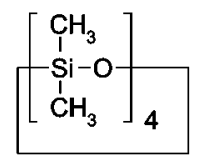

(D)

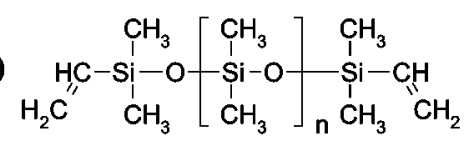

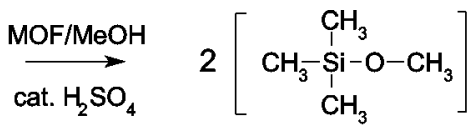
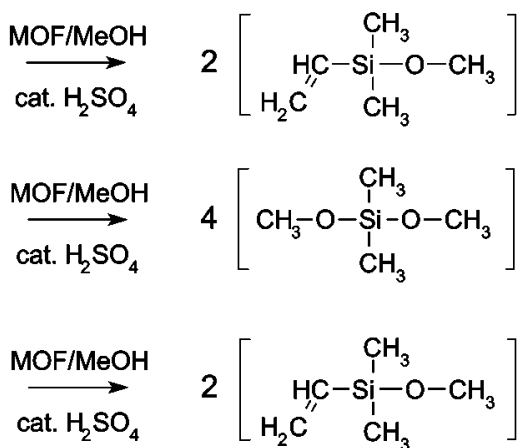

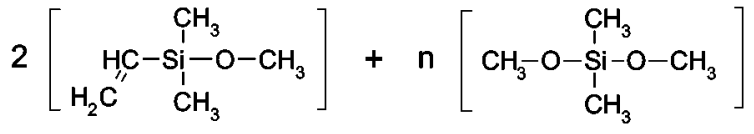

Fig. 1 Decomposition reactions by the MOF decomposition method. (A) HMDS, (B) VMDS, (C)

D4, (D) PDMS.

reported to date are insufficient for analyzing the structure and composition of silicone in detail.

Recently, the decomposition method based on the reaction between siloxane and orthoacid ester in the presence of an acid catalyst and an alcohol has received increased attention. ${ }^{10,11}$ Although a detailed reaction mechanism for this decomposition procedure is unclear, it should basically follow the format of an alkaline decomposition of the siloxane bonds, corresponding to the reverse reaction of the condensation of the alkoxysilanes. After the siloxane bonds are cleaved by the decomposition reaction, methoxysilylated siloxane monomers are generated. Because the reaction proceeds in high yield under mild acidic conditions at room temperature, this process should be a promising method to decompose silicone polymers into the constituent monomers. However, this method was originally developed mainly for material production, modification and recycling of silicone compounds. For example, the method should be useful for the re-use of the silicone materials, the removal of the polysiloxane films on some substrate, and the modification of the silicone surface by its partial degradation. In such applications, the decomposition yields of the silicone compounds were reported to be $20-60 \% .^{11}$ This fact indicates that the method is not necessarily suited for the pretreatment procedure for the quantitative analysis of silicone samples at least in the original conditions.

In this study, the decomposition conditions of the silicone materials using orthoacid were optimized in order to develop a novel procedure for quantitative analysis of silicone samples. Methyl orthoformate was used as the orthoester because of its high reactivity among various orthoesters. ${ }^{10,11}$ In addition, the decomposition products of the silicone samples by methyl orthoformate were chiefly composed of methoxysilyl groups, which are readily analyzed by GC. The optimal conditions to decompose efficiently the siloxane bond without inducing side reactions were verified using cyclic and linear siloxane oligomers and polymers with side chain methyl groups and vinyl terminals. The decomposition efficiency of the procedure was evaluated quantitatively on the basis of the observed yields of the decomposition products.

\section{Experimental}

\section{Reagents and samples}

Cyclic siloxane [octamethylcyclotetrasiloxane (D4)] and siloxane dimers (hexamethyldisiloxane (HMDS) and 1,3-divinyltetramethyldisiloxane (VMDS)) were purchased from Shin-Etsu Chemical Co., Ltd. (Tokyo, Japan). Siloxane polymer (vinyl-terminated polydimethylsiloxane (PDMS), average molecular weight was approximately 8000) was purchased from Gelest, Inc. (Morrisville, PA). Sulfuric acid (ultrahigh purity), anhydrous methanol $(\mathrm{MeOH})$, and methylorthoformate (MOF) were purchased from Kanto Chemical Co., Ltd. (Tokyo, Japan). Standard reagents for quantification such as dimethyldimethoxysilane (DMMS), dimethylmethoxyvinylsilane (VMS), and trimethylmethoxysilane (TMS) were purchased from Shin-Etsu Chemical Co., Ltd. All the reagents and the samples were used as received.

\section{Decomposition method}

Figure 1 shows the possible reactions by the MOF decomposition method of silicone samples used in this work. The siloxane $(\mathrm{Si}-\mathrm{O})$ bonds in each sample were totally cleaved and capped with methoxy groups to form the monomeric products. In order to confirm the basic decomposition pathways, low molecular weight siloxane compounds (HMDS, VMDS, D4) were first subjected to the MOF decomposition process. About $0.3 \mathrm{~g}$ of the sample compound was weighed into a glass screw bottle (volume, $20 \mathrm{~mL}$ ). Then about $30 \mathrm{mg}$ sulfuric acid, about $2 \mathrm{~g}$ of $\mathrm{MeOH}$, and about $2 \mathrm{~g}$ MOF were added as the decomposition reaction reagents. The reaction proceeded for $3 \mathrm{~h}$ at room temperature using a Teflon stirrer to uniformly mix the decomposition reaction reagents and sample. The reaction conditions were basically identical with those reported previously. ${ }^{10,11}$

As for the siloxane polymer (PDMS), about $1 \mathrm{~g}$ of the sample was weighed and placed into a round flask (volume, $50 \mathrm{~mL}$ ). Then approximately $16 \mathrm{~g}$ of MOF, $4 \mathrm{~g}$ of $\mathrm{MeOH}$, and about $30 \mathrm{mg}$ of sulfuric acid were added and the mixture was stirred. Then the round flask was connected to a condenser and heated under reflux for 12 or $30 \mathrm{~h}$ at approximately $60^{\circ} \mathrm{C}$ to accelerate the reaction because it was reported that PDMS could not be decomposed to the monomer completely at room temperature even for 2 - 22 days. ${ }^{11}$ 


\section{Sample preparation}

For the low molecular weight siloxane compounds, GC/MS samples were prepared by diluting the reaction solution after decomposition (about $100 \mathrm{mg}$ ) with $1.5 \mathrm{~g} \mathrm{MeOH}$. Similarly, ${ }^{1} \mathrm{H}$ NMR samples were prepared by diluting the reaction solution (about $100 \mathrm{mg}$ ) with $0.6 \mathrm{~mL}$ chloroform- $d$. The control GC/MS samples were prepared by dissolving $10 \mathrm{mg}$ of the original low molecular weight siloxane compound in about $1.5 \mathrm{~g} \mathrm{MeOH}$, while control samples for ${ }^{1} \mathrm{H}$ NMR were prepared by dissolving $10 \mathrm{mg}$ of the original siloxane compound in about $0.6 \mathrm{~mL}$ of chloroform- $d$.

For the silicone polymer sample, about $1 \mathrm{~mL}$ of the reaction solution was diluted with about $20 \mathrm{~mL}$ of HPLC grade chloroform and then was filtered with a PTFE filter, prior to the SEC measurement. About $100 \mathrm{mg}$ of the reaction solution was diluted with about $0.5 \mathrm{~mL}$ of chloroform- $d$ to prepare the sample for the ${ }^{1} \mathrm{H}$ NMR measurement. About $0.3 \mathrm{~g}$ of chlorobenzene was dissolved in $50 \mathrm{~mL}$ of $\mathrm{MeOH}$, and this stock solution was used as an internal standard solution for GC/MS measurements. The reaction solution mixed with the internal standard was diluted 10-fold prior to the GC/MS measurement. The control sample for SEC, which contained about $30 \mathrm{mg}$ of the undecomposed siloxane polymer diluted in about $10 \mathrm{~mL}$ of chloroform, was filtered through a PTFE filter. The GC/MS control sample was prepared by placing about $10 \mathrm{mg}$ of the undecomposed siloxane polymer in about $10 \mathrm{~mL}$ of $\mathrm{MeOH}$, while the control ${ }^{1} \mathrm{H}$ NMR sample was prepared by dissolving $10 \mathrm{mg}$ of the original siloxane polymer in about $0.6 \mathrm{~mL}$ of chloroform- $d$.

\section{Measurements}

${ }^{1} \mathrm{H}$ NMR spectra were recorded with a $600-\mathrm{MHz}$ (INOVA600) spectrometer at $293 \mathrm{~K}$ using $\mathrm{CDCl}_{3}$ as a solvent and locking agent. The spectra were obtained using a spectral width of 9615.4 Hz, acquisition time of $3.4 \mathrm{~s}$, flip angle of $45^{\circ}$, and pulse delay of $10 \mathrm{~s}$, with an accumulation of 16 scans.

GC/MS measurements were performed using an Agilent Technologies 6890GC equipped with a DB-5MS ((5\% phenyl)methyl polysiloxane, $30-\mathrm{m}$ length, $0.25-\mu \mathrm{m}$ film thickness, $0.25-\mathrm{mm}$ i.d.) capillary column and a quadrupole mass spectrometer $5973 \mathrm{~N}$ as a detector. The carrier gas was helium at a flow rate of $1 \mathrm{~mL} / \mathrm{min}$. The sample injection volume was $1.0 \mu \mathrm{L}$. Split conditions were 20:1 for low molecular weight siloxane compound and 100:1 for the siloxane polymer. The column temperature was initially set at $35^{\circ} \mathrm{C}$ for $2 \mathrm{~min}$, then programmed to $100^{\circ} \mathrm{C}$ at $5^{\circ} \mathrm{C} / \mathrm{min}$, and finally increased to $280^{\circ} \mathrm{C}$ at $20^{\circ} \mathrm{C} / \mathrm{min}$. The injector and the detector temperatures were set at 250 and $280^{\circ} \mathrm{C}$, respectively. For GC/MS detection, an electron ionization system was used with an ionization energy of $70 \mathrm{eV}$. The detected ions were recorded in the mass range $\mathrm{m} / \mathrm{z}, 50-690$ at a scan rate of $20 \mathrm{~Hz}$. Both the absolute calibration method and internal standard method were used to determine the decomposition products in the GC/MS method.

SEC measurements were performed with a Hitachi differential refractometer (L-3300), Shodex column oven (AO-50), JASCO liquid feed pump (PU-980), and Tosoh autosampler (AS-8010). The separation column was a Showa Denko GPC column K-806L ( $8.0 \mathrm{~mm}$ i.d. $\times 300 \mathrm{~mm}$ ), while the guard column was a Showa Denko K-G $(4.6 \mathrm{~mm}$ i.d. $\times 10 \mathrm{~mm})$. HPLC grade chloroform was used as the mobile phase with a flow rate of $1 \mathrm{~mL} / \mathrm{min}$. The column temperature was $40^{\circ} \mathrm{C}$, and the sample injection volume was $100 \mu \mathrm{L}$. The calibration standard materials were polystyrene standards for molecular weight 370 - 3730000 manufactured by Showa Denko.

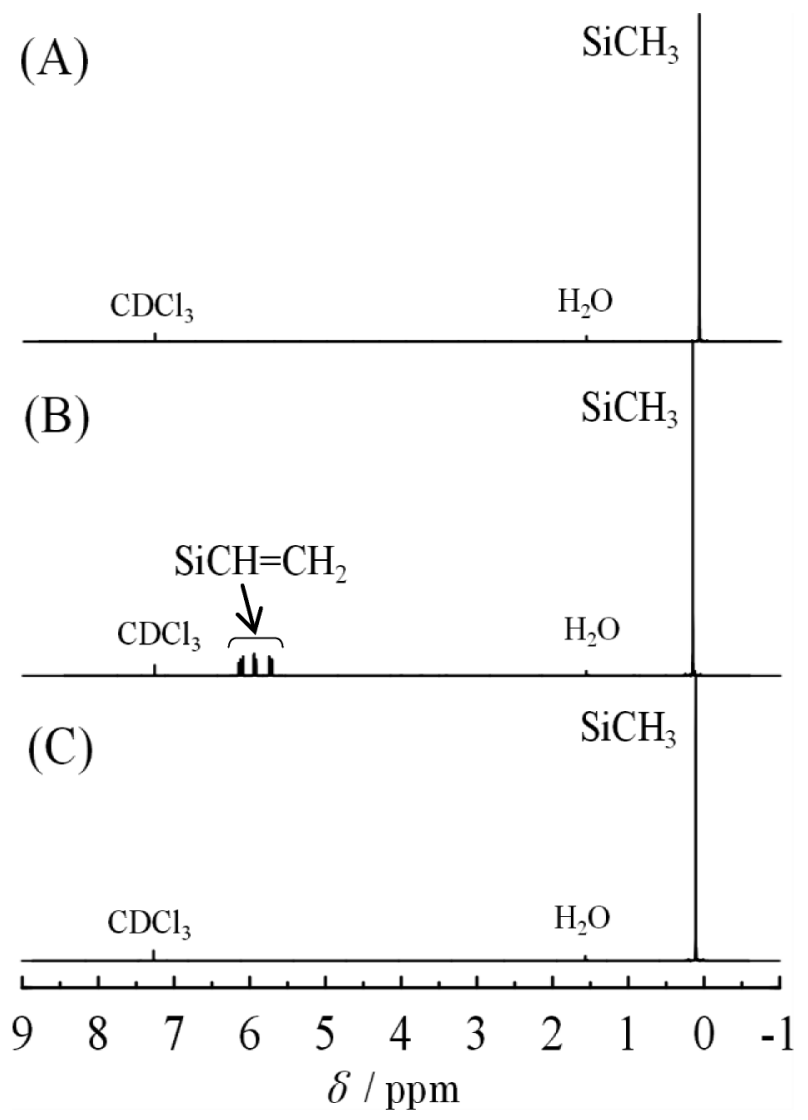

Fig. $2 \quad{ }^{1} \mathrm{H}$ NMR spectra of the original siloxane compounds before the MOF decomposition reaction. (A) HMDS, (B) VMDS, (C) D4.

\section{Results and Discussion}

Low molecular weight siloxane compounds

Figure 2 shows the ${ }^{1} \mathrm{H}$ NMR spectra of the unreacted low molecular weight siloxane compounds. Signals of methylsilyl groups in the spectra of (A) HMDS, (B) VMDS, and (C) D4 are observed at $0.06,0.15$, and $0.10 \mathrm{ppm}$, respectively. In addition, the signal of the vinylsilyl group is observed at $5.72-6.15 \mathrm{ppm}$ in the spectrum of VMDS. The slight difference in the chemical shift of the methylsilyl group for each compound should reflect that of the electron density depending on the molecular structure, which decreases in the order of HMDS with a trimethylsilyl group > D4 with a dimethylsilyl group > VMDS with a dimethylvinylsilyl group because the trimethylsilyl group has the highest electron density. Therefore, the signals were shifted downfield in this order.

Figure 3 shows the ${ }^{1} \mathrm{H}$ NMR spectra of the low molecular weight siloxane compounds after the decomposition reaction. The signals of the vinylsilyl group and methylsilyl group were observed at $5.77-6.15 \mathrm{ppm}$ and $0.11,0.19$, and $0.13 \mathrm{ppm}$, respectively for (A) HMDS, (B) VMDS and (C) D4. Signals derived from $\mathrm{MeOH}$ and $\mathrm{MOF}$, which were used as the decomposition reaction reagents, were also observed. The signals of the methoxysilyl group produced by the decomposition reaction were observed at $3.42,3.43$, and $3.50 \mathrm{ppm}$ for (A) HMDS, (B) VMDS and (C) D4, respectively. Table 1 shows the relative contents of the methylsilyl, methoxysilyl, and vinylsilyl groups, which were determined from the integral value of each signal. Although the observed contents of the methoxysilyl 


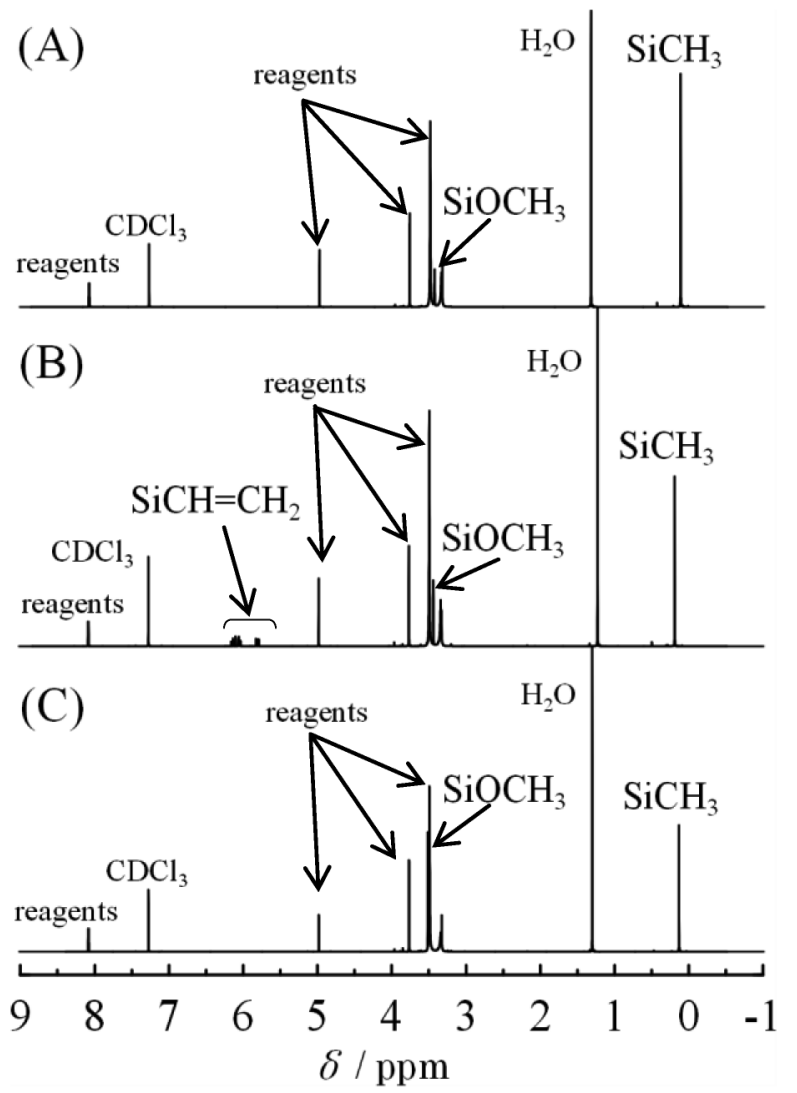

Fig. $3{ }^{1} \mathrm{H}$ NMR spectra of the siloxane compounds after the MOF decomposition reaction. (A) HMDS, (B) VMDS, (C) D4.

Table 1 Relative content of functional groups in the siloxane compounds

\begin{tabular}{lcc}
\hline Sample & $\begin{array}{c}\text { Theoretical value } \\
\mathrm{SiCH}_{3} / \mathrm{SiOCH}_{3} / \mathrm{SiCHCH}_{2}\end{array}$ & $\begin{array}{c}\text { Measured value } \\
\mathrm{SiCH}_{3} / \mathrm{SiOCH}_{3} / \mathrm{SiCHCH}_{2}\end{array}$ \\
\hline HMDS & $3.0 / 1.0 / 0.0$ & $3.0 / 1.2 / 0.0$ \\
VMDS & $2.0 / 1.0 / 1.0$ & $2.0 / 1.2 / 1.0$ \\
D4 & $1.0 / 1.0 / 0.0$ & $1.0 / 1.1 / 0.0$ \\
\hline
\end{tabular}

group were slightly larger than the theoretical value probably due to overlap with the signal of $\mathrm{MeOH}$, the contents of the functional groups after decomposition basically agrees with the theoretical values in the original siloxane compound. This result suggests that the siloxane bonds in the low molecular weight siloxane compound almost quantitatively decomposed.

Figure 4 shows the GC/MS chromatogram of the unreacted low molecular weight siloxane compound. The peaks of (A) HMDS, (B) VMDS and (C) D4 were detected at 3.3, 7.4, and $11.6 \mathrm{~min}$, respectively. Figure 5 shows the GC/MS chromatogram of the low molecular weight siloxane compound after the decomposition reaction. All of the peaks in Fig. 4 were eliminated, and the decomposition products such as TMS, VMS, and DMMS were detected at 2.1, 2.9, and $2.8 \mathrm{~min}$ for D4, VDMS, and HMDS, respectively. This result demonstrates that the low molecular weight siloxane compounds were completely decomposed into the monomeric products through the reaction shown in Fig. 1.

The decomposition products were then quantified from the corresponding peak area recorded by GC/MS measurements.

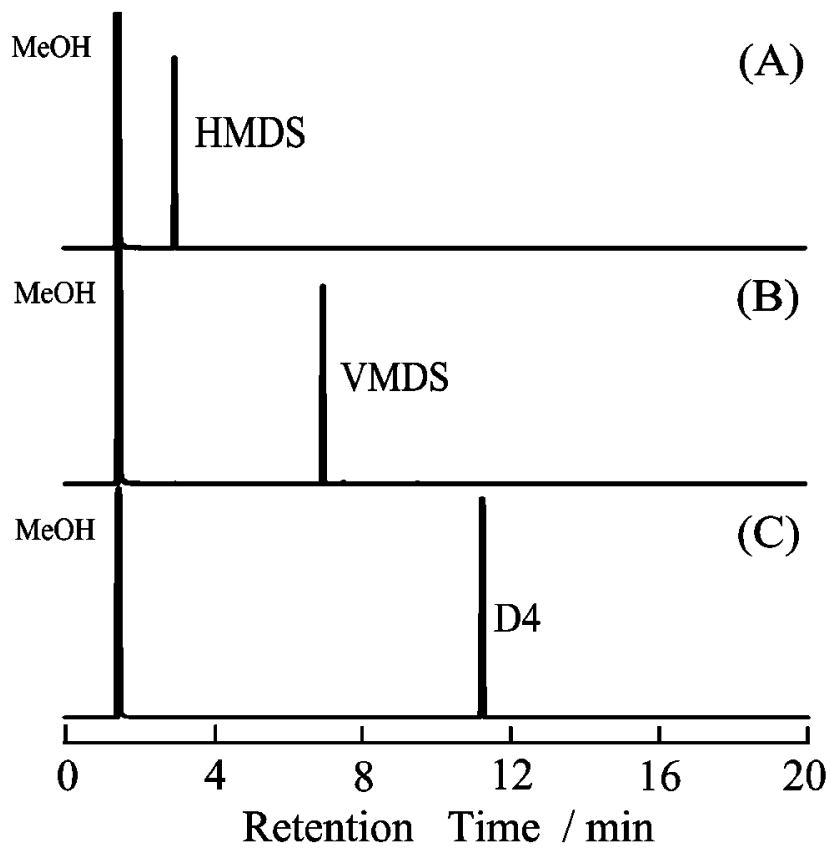

Fig. 4 GC/MS chromatograms of the original siloxane compounds before the MOF decomposition reaction. (A) HMDS, (B) VMDS, (C) D4.

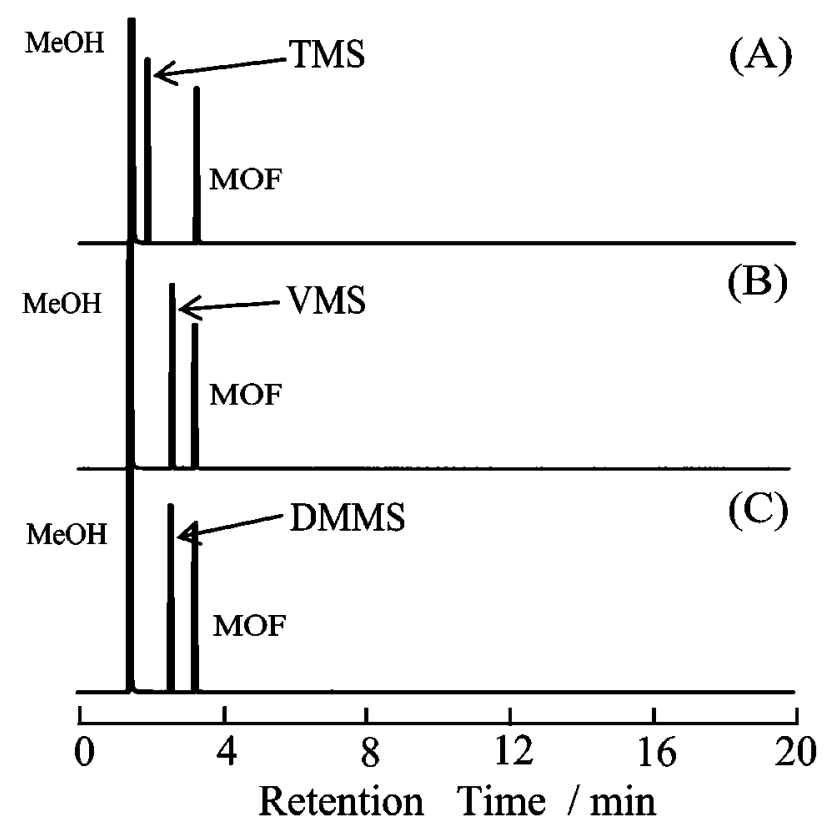

Fig. 5 GC/MS chromatograms of the siloxane compounds after the MOF decomposition reaction. (A) HMDS, (B) VMDS, (C) D4.

Table 2 shows the yields of the decomposition products. The theoretical values were calculated based on the reactions shown in Fig. 1. For each mole of HMDS (or VDMS), two moles of the decomposition product, TMS (or VMS), were generated, whereas for each mole of D4, four moles of the decomposition product, DMMS, were produced. The measured amount of the decomposition products were obtained through a comparison with the GC/MS measurements of the corresponding standard reagents. The decomposition yields of the low molecular weight siloxane compounds were thus calculated to be $99-102 \%$. 
Table 2 Yields of the MOF decomposition products of the siloxane compounds observed by GC/MS

\begin{tabular}{llcccc}
\hline Sample & $\begin{array}{c}\text { Decomposi- } \\
\text { tion } \\
\text { product }\end{array}$ & $\begin{array}{c}\text { Theoretical } \\
\text { value (g/g) }\end{array}$ & value $(\mathrm{g} / \mathrm{g})$ & $\begin{array}{c}\text { Decomposi- } \\
\text { tion } \\
\text { yield, \% }\end{array}$ & $\begin{array}{c}\mathrm{CV} \\
\text { value }\end{array}$ \\
\hline HMDS & TMS & 1.28 & 1.28 & 99.7 & 1.58 \\
VMDS & VMS & 1.25 & 1.27 & 101.7 & 0.48 \\
D4 & DMMS & 1.62 & 1.65 & 101.8 & 2.16 \\
\hline
\end{tabular}

a. For 3 repeated runs.

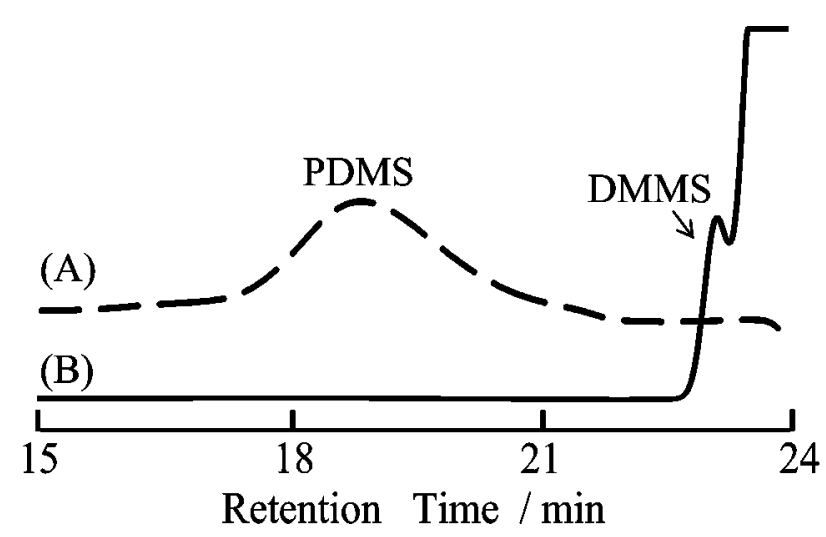

Fig. 6 SEC chromatograms of PDMS (A) before and (B) after the MOF decomposition reaction (reaction time, $30 \mathrm{~h}$ ).

These results also indicate that the low molecular weight linear and cyclic siloxane compounds with vinyl and/or methyl groups in the side chain thoroughly decomposed into the corresponding monomeric products by the MOF decomposition method.

\section{Siloxane polymer}

Figure 6 shows the SEC chromatogram of PDMS before and after the MOF decomposition reaction. The original PDMS was detected in the high molecular weight region with a peak top at $18.8 \mathrm{~min}$ as shown in Fig. 6(A). After the decomposition reaction for $30 \mathrm{~h}$, the peak of the original compound in the high molecular weight region disappeared, and a peak for the decomposition products was detected at $22.9 \mathrm{~min}$. These results suggest that the high molecular weight component of PDMS completely decomposed into much smaller compounds.

Figure 7 shows the ${ }^{1} \mathrm{H}$ NMR spectra of PDMS before (A) and after (B) the MOF decomposition reaction. In the spectrum of the original PDMS (A), signals of the terminal vinylsilyl group and the main chain dimethylsilyl group were observed at 5.72 $6.16 \mathrm{ppm}$ and $0.04 \mathrm{ppm}$, respectively. After the decomposition reaction proceeded for $30 \mathrm{~h}$, signals of the vinylsilyl group and the dimethylsilyl group were still observed at 5.66-6.06 ppm and $0.02 \mathrm{ppm}$, respectively, as shown in the enlarged spectrum of (B). In addition, a signal of the methoxysilyl group, which was generated by the decomposition reaction, was observed at $3.39 \mathrm{ppm}$. Signals derived from $\mathrm{MeOH}$ and $\mathrm{MOF}$, which are the decomposition reaction reagents, were also observed. The relative abundance of the methoxysilyl group to the methylsilyl group was obtained from the integral value of each signal; the theoretical value calculated from the ${ }^{1} \mathrm{H}$ NMR spectrum of the original PDMS before the decomposition reaction was 99.1/100.0, while the measured value in the ${ }^{1} \mathrm{H}$ NMR spectrum of PDMS after decomposition was 105.9/100.0. The measured ratio of the methoxysilyl group was slightly larger than the

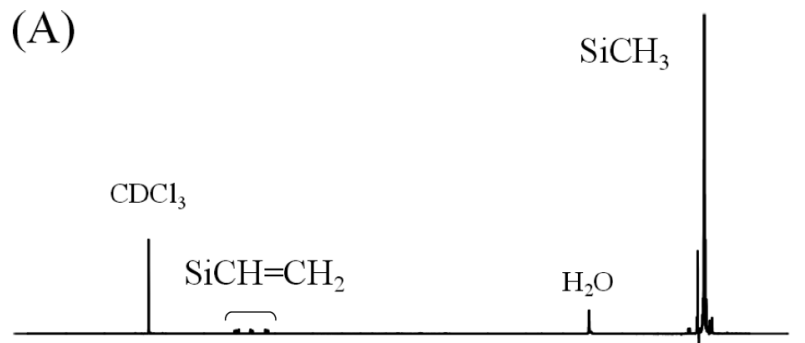

(B)

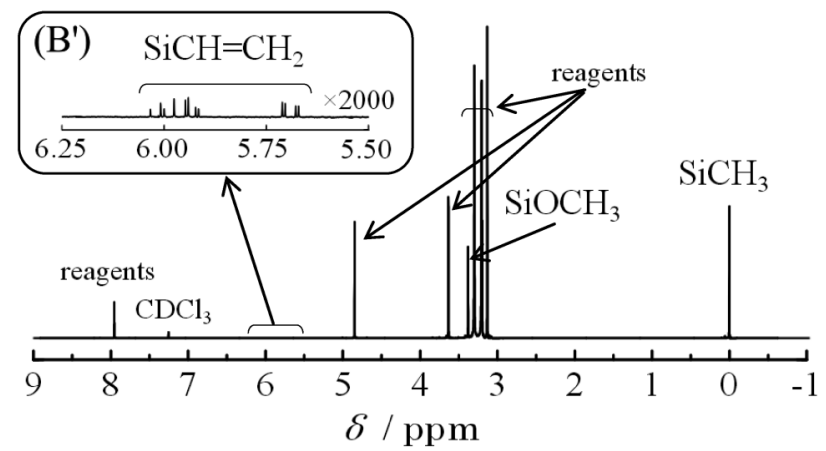

Fig. $7 \quad{ }^{1} \mathrm{H}$ NMR spectra of PDMS (A) before and (B) after the MOF decomposition reaction (reaction time, $30 \mathrm{~h}$ ); ( $\left.\mathrm{B}^{\prime}\right)$ enlarged spectrum from 5.50 to $6.25 \mathrm{ppm}$ for (B).

theoretical one due to the overlap with the $\mathrm{MeOH}$ signal; the abundance ratio agreed well with the theoretical value, indicating that the siloxane bonds of the PDMS were totally decomposed by the MOF decomposition method.

Figure 8 shows the GC/MS chromatogram of the PDMS sample before (A) and after the MOF decomposition for $12 \mathrm{~h}$ (B) and for $30 \mathrm{~h}(\mathrm{C})$. Prior to the decomposition reaction (A), no peak was detected in the chromatogram because of the high molecular weight of the original PDMS. After the decomposition for $12 \mathrm{~h}$, DMMS and some oligomeric dimethylsiloxanes, which are the decomposition products of the main chain, were clearly detected. The oligomeric products disappear after the decomposition for $30 \mathrm{~h}(\mathrm{C})$, whereas DMMS and VMS are observed in the chromatogram as the decomposition products of the main chains (DMMS) and terminals (VMS), respectively. These results demonstrate that the siloxane bond cleavages in the PDMS sample are completed by the MOF decomposition for $30 \mathrm{~h}$ but still insufficient after the decomposition for $12 \mathrm{~h}$.

Table 3 shows the observed yields of the decomposition products for $30 \mathrm{~h}$ from the PDMS sample estimated on the basis of the observed ${ }^{1} \mathrm{H}$ NMR spectrum of the original PDMS. First, the ratio of the terminal vinylsilyl group $\left(\mathrm{CH}_{2}=\mathrm{CHSi}\left(\mathrm{CH}_{3}\right)_{2} \mathrm{O}^{-}\right)$ and the main chain dimethylsilyl group $\left(-\mathrm{Si}\left(\mathrm{CH}_{3}\right)_{2} \mathrm{O}-\right)$ was calculated from the integral values of the corresponding signals in the spectrum (Fig. 7(A)), which can be correlated to the number of repeat units in a main chain. Theoretical yields of the decomposition product produced were estimated from the molecular weight of the degradation products, the number of the repeat units in the main chain and at the terminal, and the sample weight. The observed amounts of the decomposition products in the sample solution were obtained by GC/MS measurements with the calibrations using the corresponding standard reagents.

The decomposition yields of the chain end and the main chain were calculated to be about 103 and $98 \%$, respectively, indicating very high efficiencies of the MOF decomposition for $30 \mathrm{~h}$. 


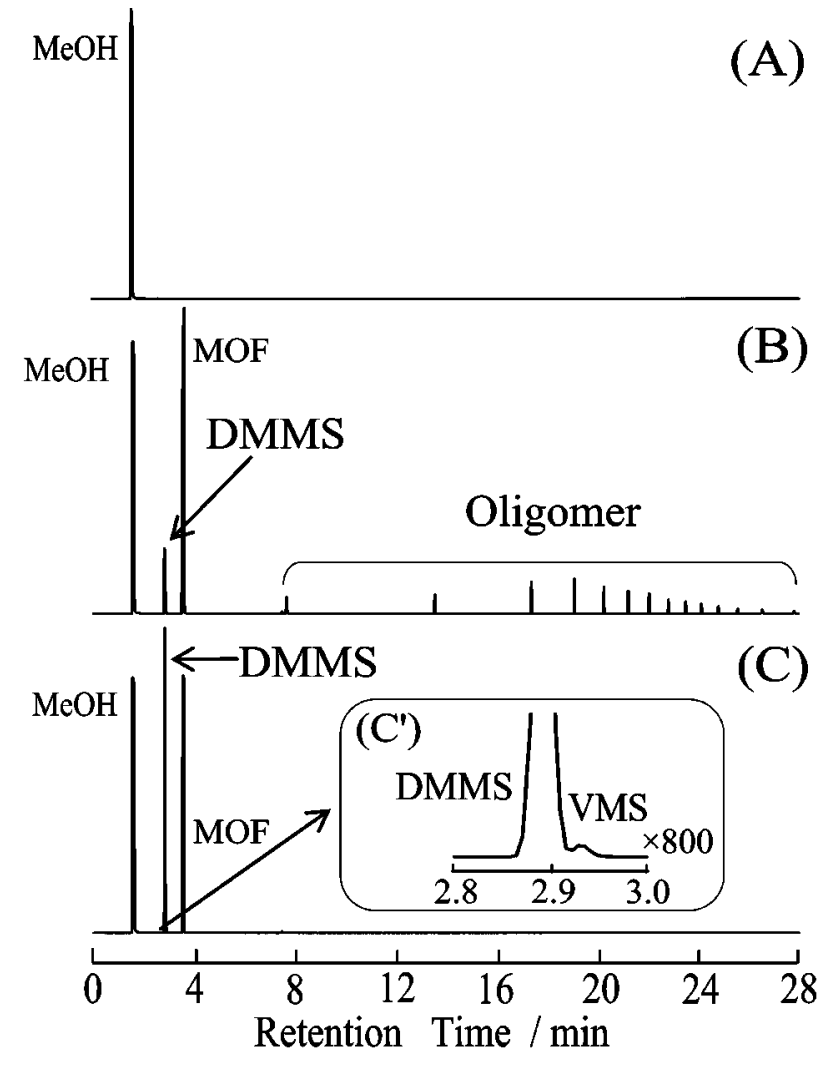

Fig. 8 GC/MS chromatograms of PDMS before and after the MOF decomposition reaction. (A) before the MOF decomposition reaction; (B) after the MOF decomposition reaction for $12 \mathrm{~h}$; (C) after the MOF decomposition reaction for $30 \mathrm{~h}$; $\left(\mathrm{C}^{\prime}\right)$ enlarged chromatogram from 2.8 to $3.0 \mathrm{~min}$ for $(\mathrm{C})$.

\begin{tabular}{llcccc}
$\begin{array}{l}\text { Table } 3 \\
\text { Yields of the products of }\end{array}$ & PDMS after & MOF \\
decomposition for $30 \mathrm{~h}$ observed by GC/MS & & \\
\hline $\begin{array}{c}\text { Decomposition } \\
\text { product }\end{array}$ & $\begin{array}{c}\text { Theoretical } \\
\text { value }(\mathrm{g} / \mathrm{g})\end{array}$ & $\begin{array}{c}\text { Measured } \\
\text { value }(\mathrm{g} / \mathrm{g})\end{array}$ & $\begin{array}{c}\text { Decomposition } \\
\text { yield, } \%\end{array}$ & $\begin{array}{c}\mathrm{CV} \\
\text { value }^{\mathrm{a}}, \%\end{array}$ \\
\hline DMMS & 1.58 & 1.55 & 97.9 & 1.40 \\
VMS & 0.029 & 0.030 & 102.5 & 2.89 \\
\hline
\end{tabular}

a. For 3 repeated runs.
These results indicate that even the siloxane polymer with a large molecular weight decomposes into the corresponding monomeric products possessing vinyl and/or methyl groups with high decomposition yields.

\section{Conclusions}

It was demonstrated that the MOF decomposition method can decompose not only the low molecular weight linear and cyclic siloxane compounds but also the siloxane polymer into the corresponding monomeric products possessing vinyl terminals and side chain methyl groups under mild acidic conditions around room temperature. The observed decomposition yields were almost quantitative even for the siloxane polymer under the optimized conditions. This fact suggests the MOF decomposition method is a promising technique to characterize silicone materials in detail. Further research is currently in progress to apply this method for the structural and compositional analyses of various silicone materials with other side chain functional groups and/or cross-linking points.

\section{References}

1. S. Kaneko, Y. Tsushima, Y. Yoshida, and M. Kayano, Bunseki Kagaku, 1986, 35, 189.

2. Y. Zenko, M. Noto, and R. Tanaka, Kateiyaku Kenkyu, 1988, 7, 48.

3. S. Fujimoto, H. Ohtani, and S. Tsuge, Fresenius' Z. Anal. Chem., 1988, 331, 342.

4. S. Fujimoto, H. Ohtani, K.Yamagiwa, and S. Tsuge, J. High Resolut. Chromatogr., 1990, 13, 397.

5. A. Ballistrere, D. Garozzo, and G. Montaudo, Macromolecules, 1984, 17, 1312.

6. N. Grassie and R. S. Bettie, Polym. Degrad. Stab., 1984, 7, 231.

7. G. W. Heylmun and J. E. Pikula, J. Gas Chromatogr., 1965, 266.

8. D. D. Schlueter and S. Siggla, Anal. Chem., 1977, 49, 2343.

9. K. J. Haken, N. Harahap, and P. R. Burford, J. Chromatogr., 1990, 500, 367.

10. R. Tsuji, Japan Patent 3529854.

11. T. Hirose, R. Tsuji, and K. Oouchi, Japan Patent 3529858. 